\title{
Theoretical Prediction for Band Gap of Semiconducting Nanoparticles
}

\author{
Sachin ${ }^{1}$, Brijesh Kumar Pandey ${ }^{2, *, \mathbb{D}, \text { Ratan Lal Jaiswal }}{ }^{3}$
}

In the present study, a well-established relation between the band gap of low dimensional solid and its cohesive energy has been used to calculate the band gap variation at nano level. The different thermodynamical models proposed for the cohesive energy do not consider the unsaturated bonds of surface atoms, the shape of the nanoparticle and the packing density of the corresponding crystal simultaneously. Extending the bond energy model, a simple theoretical model for the cohesive energy has been proposed which incorporate all the above-mentioned properties simultaneously and hence getting a more comprehensive relation between the band gap and the characteristics of the nanoparticle. We have computed bandgap of compound semiconducting nanosolids $\mathrm{ZnE}$ and $\mathrm{CdE},(\mathrm{E}=\mathrm{S}, \mathrm{Se}, \mathrm{Te})$ in different shapes. It is found that band gap expands as the particle size decreases and the shape deviates more from spherical one.

A close agreement between our calculated results and the available experimental data validates the present theoretical model. The present expression of Band gap of Nanosolids is potentially applicable for those materials whose experimental data are not available.

\section{Introduction}

As a result of size reduction up to the nanoscale, the physical properties of nanomaterials change in a very significant way, which has provided tremendous opportunities giving way to new technological revolution. It has become possible to fabricate materials with tunable properties [1-3]. The optoelectronic properties such as the band gap of semiconductors can be engineered in such a way that suits our requirement because it can be altered by changing the size at nano-level. The optoelectronic devices can be manufactured utilizing the tunability on account of size variation of nanoscaled semiconductors. The tunability of the band gap has shown fantastic opportunities as it empowers nanosized semiconducting materials such as $\mathrm{Si}$, Ge, $\mathrm{ZnE}(\mathrm{E}=\mathrm{S}, \mathrm{Se}, \mathrm{O})$ and $\mathrm{AlN}$, GaN to be employed in many optical devices such as lasing diodes, high speed FETs, Solar cells etc. [4,5]. When the size of the material is reduced, two fundamental nano-scale effects arise-surface effects and spatial confinement effects. Spatial confinement effects are quantum mechanical in nature and become appreciable when a characteristic limit is reached. The

\footnotetext{
1'Department of Physics, VBS Government Degree College Campierganj, Gorakhpur 273158, India

${ }^{2}$ Department of Physics \& Material Science, MMM University of Technology, Gorakhpur 273010, India

${ }^{3}$ Department of Physics, Government Degree College, Dhadha Bujurg, Hata, Kushinagar 274207, India

*Corresponding author:

E-mail: bkpmmmec11@gmail.com
}

nature of chemical bonds and the property decide the critical limit. Because of quantum confinement effects, the excitons (electron-hole pairs) are formed and consequently the band gap increases with decrease of the size of the nanoparticle [4].

To explain the band gap tunability, a lot of studies have been done and different theoretical models have been proposed [6-24]. Among them, the thermodynamic models, however, do not incorporate the surface effect especially the dangling bond, arrangement of atoms in their crystalline structure, i.e., packing factor of the structure and the deviations from the spherical shape of the nanoparticle, i.e., the shape factor simultaneously. Since the energy is a fundamental aspect of materials [25], a careful thermodynamic analysis of energy at nano-scale is a useful way to estimate size and shape dependent band gap. Utilizing a well-established relationship of the energy band gap with the cohesive energy model of crystal, we have proposed a new model for cohesive energy of nanosolids by extending the Bond Energy Model [26] to include the packing factor also. Thus, our proposed model incorporates all the three concepts - the dangling bond concept, the packing and the shape factors of the nanoparticle, simultaneously in the size-dependent cohesive energy expression based on bond energy model (BEM). Using a modified expression for cohesive energy at nanoscale, a thermodynamic model for the band gap variation is constructed in this work.

The theoretical model is used to calculate the band gap variation of $\mathrm{ZnS}, \mathrm{ZnSe}, \mathrm{ZnTe}, \mathrm{CdS}, \mathrm{CdSe}$ and CdTe compound semiconducting nanosolids in different shapesspherical as well as non-spherical such as tetrahedral, hexahedral and octahedral. The comparison of our

DOI: 10.5185/aml.2021.15700 


\section{Advanced Materials Letters https://aml.iaamonline.org}

simulated data with the experimental one is made graphically. All the evidences depicted in the graphs suggested that the size-reduction and the deviations from sphericity of nanostructures expand the band gap in nanorange. Our model can be applied in the prediction of the band gap of the semiconducting nanomaterials where the experimental data is not available.

\section{Mathematical model}

The limited dimensions of the nanoparticle lead to quantum confinement effects [27] which become important when the size decreases below a certain critical limit, given by in case of optoelectronic properties, Exciton Bohr diameter. This Exciton Bohr diameter depends on the material and can vary from $1 \mathrm{~nm}$ to more than $100 \mathrm{~nm}$. If the size of the semiconductor nanocrystal approaches the size of exciton, the spatial confinement of charge carriers increases their energy which is manifested in the expansion of the band-gap energy.

The crystalline band structure can be explained by the nearly-free-electron approach according to which the band gap $E_{G}$ is related to the crystalline potential $V$, which is dependent on the total number of constituent atoms and the inter-atomic interaction of the crystal. According to this approach, the first Fourier coefficient of the crystalline potential determines the energy gap, i.e., $E_{\mathrm{G}}=2\left|V_{1}\right|[\mathbf{2 8}]$. Thus, any change in the crystalline field should be reflected into the band gap. If the band gap variation $\Delta E_{\mathrm{G}}=E_{G}(D)-E_{G}(\infty)$ is supposed to be linearly dependent on the change in the crystal field $\Delta V=V(D)$ - $V(\infty)$, where $E_{G}(D)$ and $V(D)$ represent the size-dependent band gap and the size-dependent crystalline field at nano-level respectively, we have

$$
\frac{\Delta E_{G}(D)}{E_{G}(\infty)}=\left|\frac{\Delta V(D)}{V(\infty)}\right|
$$

Like crystalline potential $V$, the atomic cohesive energy $E_{C}$ is also a measure of total inter-atomic interactions. Thus, we can write $E_{C} \propto V$ and hence $\Delta E_{C}(D)$ $\propto \Delta V(D)[29]$ and consequently

$$
\begin{gathered}
\qquad E_{G}(D)=\left[\delta \frac{4 \alpha d}{\eta D}\right] E_{G}(\infty) \\
\frac{\Delta E_{G}(D)}{E_{G}(\infty)}=\left|\frac{\Delta V(D)}{V(\infty)}\right|=\left|\frac{E_{C}(D)-E_{C}(\infty)}{E_{C}(\infty)}\right| \\
\text { So we get } \quad \frac{\Delta E_{G}(D)}{E_{G}(\infty)}=1-\frac{E_{C}(D)}{E_{C}(\infty)}
\end{gathered}
$$

The above expression provides a promising gateway to obtain the nano-range band gap variation $\Delta E_{G}(D)$ value if nanocrystalline cohesive energy $E_{C}(D)$ is found, since both $E_{G}(\infty)$ and $E_{C}(\infty)$ for bulk materials usually remain constants. The expression for cohesive energy of a lowdimensional solid can be obtained using bond energy model (BEM) according to which a nanosolid can be considered as a core-shell entity [26].
In the BEM, the cohesive energy of a nanoparticle is the sum of the contributions of the interior atoms as well as the atoms responsible for the outer layer. The core atoms maintain bulk-like behaviour, whereas the shell atoms having less coordination number contribute a reduced cohesive energy. Thus, the cohesive energy of a nanosolid consisting of $n$ atoms in total and $N$, surface atoms is

$$
E_{n, C}(D)=(n-N) E_{C}(\infty)+N E_{S}
$$

where $E_{\mathrm{C}}(\infty)$ is the cohesive energy of a core- atom and $E_{S}=(1-\delta) E_{\mathrm{C}}(\infty)$ is that of a surface atom; $\delta$ is a quantity known as relaxation factor, describing the dangling bonds of an atom on the surface and range $0 \leq \delta<1$. Thus, the cohesive energy per atom of a nanosolid is found as

$$
E_{C}(D)=\left(1-\frac{\delta N}{n}\right) E_{C}(\infty)
$$

Now the cohesive energy of the nanoparticle $E_{\mathrm{C}}(D)$ can be cast into an expression containing some characteristic quantities pertaining to the nanoparticle.

Because a low-dimensional solid may have nonspherical shapes also, a shape factor $(\alpha)$ must be introduced to account the shape variations. If the surface area of a nonspherical nanoparticle is $S^{\prime}$ and that of a spherical one with an equal volume as the nonspherical nanoparticle be $S$, the shape factor $\alpha$ is defined as $\alpha=\left(S^{\prime} / S\right)$. For a nanoparticle having nonspherical shape, the volume is $\left(\pi D^{3} / 6\right)$ i.e., same as that of the spherical one, and an atom of diameter $d$ has its volume is $\left(\pi d^{3} / 6\right)$. Since crystals have voids between the constituents, it is necessary to consider the packing factor $(\eta)$ also while calculating the number of atoms within the nanoparticle. This factor $(\eta)$ can be determined by calculating the volume of all atoms in a unit cell and dividing this by the volume of the unit cell $[\mathbf{3 0 , 3 1}]$, i.e. $\eta=V_{\text {all atoms }} / V_{\text {cell }}$. Now the total number of the atoms within the nanoparticle $(n)$ equals the volume of the voidless nanoparticle divided by that of an atom, i.e., $n=\eta\left(D^{3} / d^{3}\right)$. The surface enclosed by a nonspherical nanoparticle is $\left(\alpha \pi D^{2}\right)$ and the contribution to it by a surface atom being $\left(\pi d^{2} / 4\right)$, the number of the atoms forming the surface $N=4 \alpha\left(D^{2} / d^{2}\right)$. Thus, we get $[\mathbf{3 1}, 32]$

$$
\frac{N}{n}=\frac{4 \alpha d}{\eta D}
$$

The CE of per atom becomes

$$
E_{C}(D)=\left(1-\delta \frac{4 \alpha d}{\eta D}\right) E_{C}(\infty)
$$

Finally, the equation (1) tells that the band gap variation due to the size and shape variation is

$$
\Delta E_{G}(D)=\left[\delta \frac{4 \alpha d}{\eta D}\right] E_{G}(\infty)
$$

The above equation can be applied to calculate the band gap variation of the nanoparticles having different shapes i.e., spherical, octahedral, hexahedral and tetrahedral. 


\section{Advanced Materials Letters https://aml.iaamonline.org}

\section{Results and discussion}

In order to validate our model for the band gap variation, we have selected the direct band gap semiconducting materials of group II-VI, i.e., $\mathrm{ZnE}$ and $\mathrm{CdE},(\mathrm{E}=\mathrm{S}, \mathrm{Se}, \mathrm{Te})$. The input data for these compounds are given the Table 1 along with their calculated packing factor $(\eta)$ for zinc blende structure and bonded covalently. The relaxation factor has the permissible range $0 \leq \delta<1$. The shape factor $(\alpha)$ for different nonspherical shapes is given in the Table 2.

Table 1. Input data for selected materials [1].

\begin{tabular}{ccccc}
\hline Sr.No & Compound & $\boldsymbol{E}_{\boldsymbol{G}}(\boldsymbol{\infty}) \mathbf{( e V )}$ & $\boldsymbol{d}(\mathbf{n m})$ & $\boldsymbol{\eta}$ \\
\hline 1 & $\mathrm{ZnS}$ & 3.68 & 0.234 & 0.357 \\
2 & $\mathrm{ZnSe}$ & 2.7 & 0.2455 & 0.354 \\
3 & $\mathrm{ZnTe}$ & 2.26 & 0.264 & 0.356 \\
4 & $\mathrm{CdS}$ & 2.49 & 0.252 & 0.375 \\
5 & $\mathrm{CdSe}$ & 1.75 & 0.262 & 0.371 \\
6 & $\mathrm{CdTe}$ & 1.43 & 0.2805 & 0.362 \\
\hline
\end{tabular}

Table2. Input Data for Selected shapes [26].

\begin{tabular}{ccc}
\hline Sr.No. & Shape & Shape Factor $(\boldsymbol{\alpha})$ \\
\hline 1 & Spherical & 1 \\
2 & Octahedral & 1.18 \\
3 & Hexahedral & 1.24 \\
4 & Tetrahedral & 1.49 \\
\hline
\end{tabular}

Figs. 1 to 6 depict the energy gap-variation along with the experimental results available [14]. In these graphical representations of computational results, we have taken the relaxation factor within the permissible range. The energy band gap variations of the semiconductor nanoparticles $\mathrm{ZnS}, \mathrm{ZnSe}, \mathrm{ZnTe}, \mathrm{CdS}, \mathrm{CdSe}$ and CdTe with respect to size and shape have been calculated using Eq. (4).

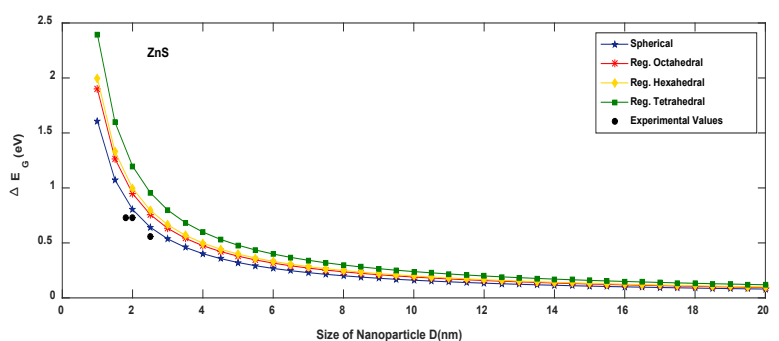

Fig. 1. Band Gap variation of $\mathrm{ZnS}$ nanoparticle, $(\bullet)$ denotes experimental values [14].

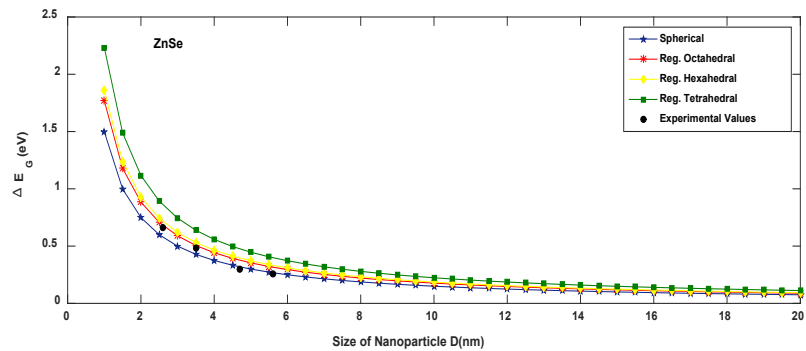

Fig. 2. Band Gap variation of $\mathrm{ZnSe}$ nanoparticle, $(\bullet)$ denotes experimental values [14].

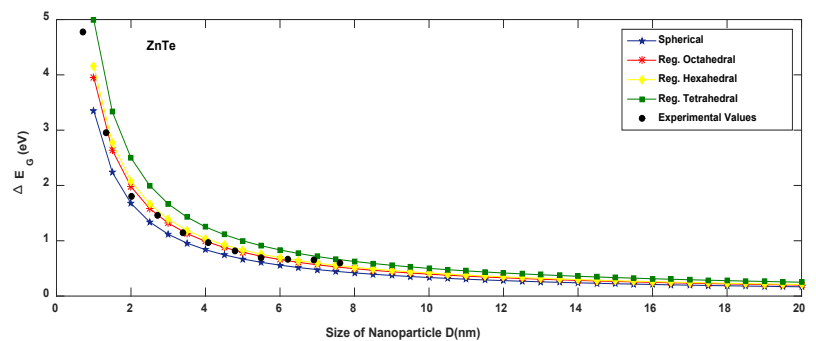

Fig. 3. Band Gap Variation of ZnTe nanoparticle, $(\bullet)$ denotes experimental values [14].

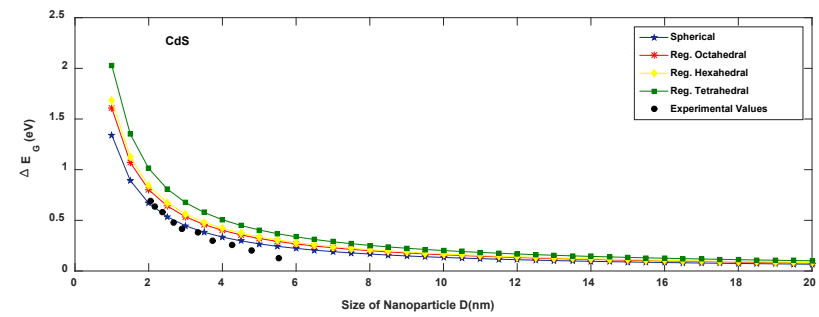

Fig. 4. Band Gap Variation of CdS nanoparticle, $(\bullet)$ denotes experimental values [14]

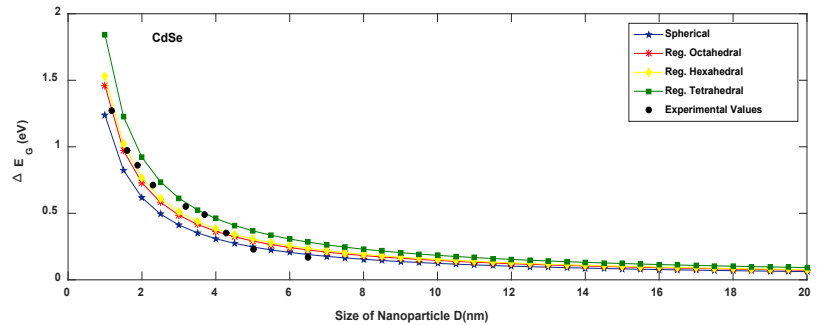

Fig. 5. Band Gap variation of CdSe nanoparticle, $(\bullet)$ denotes experimental values [14].

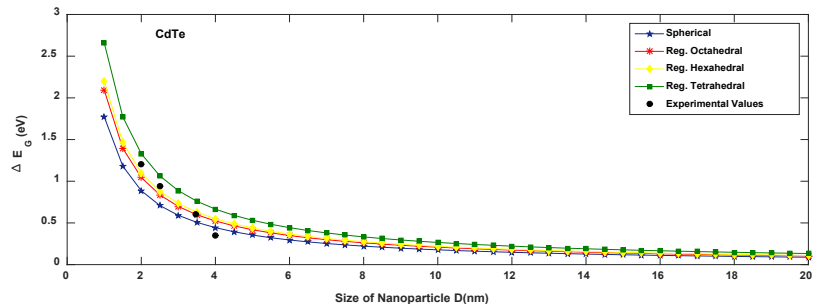

Fig. 6. Band Gap Variation of CdTe nanoparticle, $(\bullet)$ denotes experimental values [14].

It is to be noted from these graphical representations that though the band gap expands when the size of the nanoparticles is reduced, the expansion is appreciable only below $10 \mathrm{~nm}$. The figures also show that the band gap of the particles is affected by the shape of the material too, the tetrahedral shape being more expansionist than other shapes but again the energy gap-variation with shape is noticeable for small sizes only. The expansion of the band gap can be clarified by the spatial confinement effects. For a semiconducting crystal, electronic excitations known as exciton, are delocalized within the crystal. When the size of the semiconductor becomes comparable to the exciton Bohr diameter, quantum confinement effects arise. When the size of the nanoparticle becomes smaller than that of the exciton Bohr diameter, the exciton ceases to be exist and the 


\section{Advanced Materials Letters https://aml.iaamonline.org}

electron and the hole are confined separately like a particle in quantum-box situation, resulting in the band-gap expansion. As for as the shape dependence is concerned, we see that tetragonal shape expands the gap more. The observed relationship between shape and gap can be attributed to surface effects rather than spatial confinement. Some small deviations from the experimental data suggest that more insight is needed to fully explain them. It is however, observed that the expansion of the band gap with the reduction of size shows the same trend for the selected compounds which validates our model incorporating the dangling of surface atoms, voids within the crystal and the shape of the material simultaneously. Hence the tuning of band gap expansion depends on shape, size, and packing factor of the semiconductor nanomaterials. The close similarity between the predicted values of the energy gap in the nano-range with available data shows that our model for cohesive energy can be used to predict more physical properties such as dielectric function, refractive index, electrical and thermal conductivities, magnetic properties etc of nanocrystals which may be more fruitful to fabricate nanoscale devices finding applications in the service of human society.

\section{Conclusion}

The nearly free electron model is used to relate the crystal field and the band gap; and the cohesive energy of nanosolid to the crystal field. Our proposed model incorporating the size, shape, relaxation factor of surface particles and the packing of the nanomaterial, all taken together, modulate the band gap of the nanosolids in accordance with the experimental data. We found that the reduction in the size of semiconductor Nanosolids expands the gap and the larger deviations from the sphericity produce more expansion at nano-level. The observable deviations from the experimental data might be explained by, among other possibilities, exploring the impact of sizereduction and shape of the materials on the packing factor. Thus, our proposed model for Band gap of Nanosolids is potentially applicable for those materials whose experimental data are not available.

\section{Conflicts of interest}

There are no conflicts to declare.

\section{Keywords}

Nanosized compound semiconductors, quantum confinement, energy gap, cohesive energy, shape factor, dangling bond.

\section{Received: 21 March 2021}

Revised: 15 June 2021

Accepted: 15 July 2021

\section{References}

1. Poole Charles, P.; Owens, Frank Jr.; Introduction to Nanotechnology; 2nd Edition, John Wiley and Sons, UCC Press, Delhi India, 2008

2. Kittel, C.; Introduction to Solid State Physics; 7th Edition, John Wiley and Sons, Sonepat Haryana, India, 2008

3. Roduner, E.; Chem. Soc. Rev., 2006, 35, 583.

4. Smith, A. M.; Nie, S.; ACC Chem. Res., 2010, 190, 43.
5. Canham, L.T.; Appl. Phys. Lett., 1990, 57, 1046.

6. Yang, C. C.; Li, S.; J. Phys. Chem. C, 2008, 112, 2851.

7. Yang, C. C; Jiang, Q; Mater. Sci. Eng. B, 2006, 131, 191.

8. von Grunberg, H. H.; Phys Rev B, 1997, 55, 2293.

9. Wang, Y.; Ouyang, G; Wang, L.L.; Tang, L.M.; Tang, D.S.; Sun, C.Q.; Chemical Physics Letters, 2008, 463, 383.

10. Brus, L.E.; J. Chem. Phys., 1984, 80, 4403.

11. Brus, L.; J. Phys. Chem., 1986, 90, 2555.

12. Bulutay, C.; Phys Rev B., 2007, 76, 205321.

13. Chen, Y.; Li, J.; Yang, X.; Zhou, Z.; Sun, C.Q.; J. Phys. Chem. C,2011, 115, 23338.

14. Li, J.; Wang, L.; Physical Review B, 2005, 72, 125325.

15. Li, M.; Li, J.C.; Materials Letters, 2006, 60, 2526.

16. Li, M.; Li, J.C.; Jiang, Q.; J. Mod. Phys. B, 2010, 24, 2297.

17. Sapra, S.; Sharma, D.D.; Physical Review B, 2004, 69, 125304.

18. Sun, C.Q.; Tay, B.K.; Zeng, X.T.; Li, S.; Chen, T.P.; Zhou, J.; Bai, H.L.; Jiang, E.Y.; J. Phys., Condens. Matter, 2002, 14, 7781.

19. Wang, Y; Ouyang, G.; Wang, L. L.; Tang, L.M.; Tang, D. S.; Sun, C.Q.; Chemical Physics Letters, 2008, 463, 383.

20. Wilson, H. F.; McKenzie-Sell, L; S. Barnarda, A. S.; J. Mater. Chem. C, 2014, 2, 9451.

21. Yang, C.C.; Li, S.; J. Phys. Chem. C,2008, 112, 2851-2856.

22. Yang, Chun Cheng; Mai, Yiu-Wing; Materials Science and Engineering $R, \mathbf{2 0 1 4}, 79,1$

23. Yofee, A.D.; Advances in Physics, 1993, 42, 173.

24. Patel, G.R.; Pandya, T.C.; Int. J. Sci. Res. in Physics and Applied Sciences, 2018, 6, 37

25. Safaei, A.; Philos. Mag., 2011, 91, 1509

26. Qi, W.H.; Accounts of Chemical Research, 2016, 49, 1587.

27. Wang, Y.; Herron, N.; The Journal of Physical Chemistry, 1991, 95, 525 .

28. Sun, C.Q.; Chen, T.P.; Tay, B.K.; Li, S.; Huang, H.; Zhang, Y.B.; Pan, L.K.; Lau, S.P.; Sun, X.W.; J. Phys. D Appl. Phys., 2001, 34, 3470

29. Xiao, H.J.; Li, H.; Chang, L.; Zhang, H.X.; Xu, H.C.; Materials Chemistry and Physics, 2016, 181, 1 .

30. Qu, Y.D.; Liang, X.L.; Kong, X.Q.; Zhang, W.J.; Physics of Metals and Metallography, 2017, 118, 528

31. Sachin, Pandey, B.K.; Jaiswal, R.L.; Adv. Sci. Eng. Med., 2020, 12 27.

32. Jaiswal, R.L.; Pandey, B.K.; SN Applied Sciences, 2021, 3,466.

\section{Authors biography}

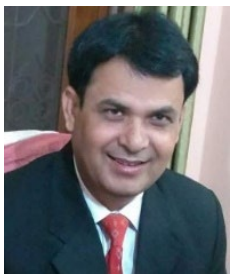

Prof. B.K. Pandey is Professor and Head in the Department of Physics and Material Science, at Madan Mohan Malaviya University of Technology, Gorakhpur, India. His research interests are in the areas of thermophysical properties of nanomaterials, thermal conductivity of nanofluids and solar cell. He has published more than 50 research papers in the peer reviewed research journals of international repute. He has also authored 18 books published from international publishers like Cengage learning and Pearson.

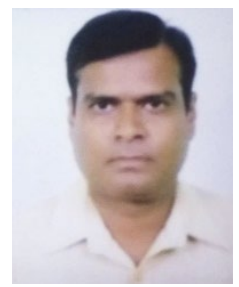

Sachin is working as an Assistant Professor in the Department of Physics, at Veer Bahadur Singh Government Degree College, Campierganj Gorakhpur, India and pursuing his $\mathrm{Ph} . \mathrm{D}$. under the supervision of Prof. B. K. Pandey on the title "Studies on Physical Properties of Metallic and Semiconducting Nanomaterials".

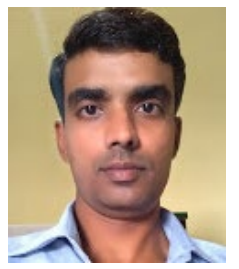

Ratan L Jaiswal is working as an Assistant Professo in the Department of Physics, at Government Degree College, Hata Kushinagar, India and pursuing his Ph.D. under the supervision of Prof. B. K. Pandey on the title "Studies on Thermophysical Properties of Nanomaterials". 